\title{
La question de la subalternité chez Gayatri Chakravorty Spivak
}

\author{
Hervé Ondoua \\ Université de Ngaoundéré
}

\section{Résumé}

Les subalternes peuvent-elles parler? C'est à cette question que s'attèle à répondre notre communication à partir d'une approche marxiste propre à Spivak. En empruntant la méthode déconstructive, il s'agit pour Spivak de résister à la traduction et à tentation de la «reconnaissance du tiers-monde par assimilation ». Aussi pour Spivak comme pour Derrida de s'interroger sur la manière d'accueillir l'Autre comme absolument étranger, sans le soumettre à la violence de la traduction, de la première question, qui es-tu ? Il ne s'agit plus de rendre invisible la pensée ou le sujet pensant, mais bien au contraire de faire ressortir l'ethnocentrisme. Le risque est toujours de se «reterritorialiser» au sein du langage hégémonique impérialiste sur un essentialisme. Il faut donc une réécriture de l'impulsion structurale utopique qui fait « délirer la voix intérieure qui est la voix de l'autre en nous ». Il s'agit pour Spivak de déconstruire, en tant qu'intellectuelle postcoloniale et décolonialiste, le concept de «femme du Tiersmonde », de désapprendre c'est-à-dire se poser en situation de recul par rapport à la manière dont elle a pu être formée comme un objet. Dès lors nous pouvons nous interroger: Quel est l'enjeu d'une philosophie qui milite pour l'acentrique épistémologique ? Cette nouvelle orientation du discours ne permet-elle pas de sortir des concepts monolithiques utilisés dans les sciences sociales pour parler des subalternes ? À partir d'une approche herméneutique, nous essayerons d'analyser la pensée de Spivak.

Mots-clés : Subalterne, colonisation, déconstruction, reconnaissance, éducation 
Parmi les objectifs que s'est assigné l'historiographie telle que développée par les Subaltern Studies, il y a l'idée de comprendre le processus de formation indienne (Francesco Fistetti, 2009 : 37). À partir de la méthode immanentiste, il est question, pour nous, de revisiter la pensée des auteurs comme Spivak qui ont influencé ce processus. Notre objectif est de montrer les enjeux de l'entreprise de critique de la rationalité occidentale telle que développée par Spivak. Quelles sont les thèses déployées par Spivak pour rendre au subalterne sa propre voix ? Telle est notre problématique centrale. Pour rappel, les ouvrages des auteurs comme Ramachandra Guha (2002), Spivak (2009), etc., peuvent être considérés comme un «acte d'accusation contre cette historiographie officielle, qui offre enfin l'occasion d'entendre la voix des classes subalternes » (Francesco Fistetti, 2009 : 37). L'histoire indienne comme celle des peuples colonisés ont été analysées et écrites par les colonisateurs. Cette analyse dénature le plus souvent la pensée de ces peuples. Redonner la voix aux subalternes est la mission que s'octroie Spivak. Subalternes est synonyme ici de marginalité ou de classes opprimées. Qui parle et qui agit ? Ce sont les interrogations que se pose Spivak. En empruntant l'approche déconstructiviste derridienne, Spivak remet en question cette lecture et cette vision occidentale. La déconstruction relit à nouveau les récits de l'Occident et ceux de la décolonisation non comme des essences mais comme des effets historiques. Ses récits ont été écrits par des colonisateurs. Il est donc question de redonner la parole aux subalternes afin de restituer leur propre histoire. Tel est l'enjeu de l'Orientalisme. L'orientalisme se définit dans ce sens comme « une archive de savoirs [...] concernant les peuples non européens » (Ibid. : 27). Cette archive constitue la vision que l'Occident a projetée sur les peuples colonisés. 
L'évocation de l'orientalisme n'est pas fortuite. L'orientalisme de Saïd a influencé plusieurs auteurs comme Gayatri Spivak. L'auteur de l'œuvre Les subalternes peuvent-elles parler? fonde sa théorie critique à partir des lectures de Saïd, notamment de ses archives qui sont le reflet des stéréotypes des colonisateurs. Aussi en « leur imposant, d'un seul et même geste, le "savoir" et le "pouvoir", l'Occident a fixé les traits de la subalternité », et, par conséquent, « défini le statut d'"une race subalterne qui jouit du gouvernement d'une race qui connait, qui sait ce qui est juste pour elle encore mieux qu'elle-même » (Ibid. : 53). C'est la raison pour laquelle Spivak partage la même idée avec les auteurs des Subaltern Studies de reconsidérer l'historiographie coloniale de l'Inde «à partir de la perspective de la chaine discontinue des révoltes paysannes pendant l'occupation coloniale » (Gayatri Spivak, 2009a : 44). Précisons avec Jean-Marc Moura que la théorie postcoloniale et par ricochet des Subaltern Studies rendent le plus souvent compte de la colonisation britannique de l'Inde, de l'Afrique ou du Proche-Orient. Pour rappel, Jean-Marc Moura affirme que « les Français ont pratiqué une politique d'assimilation culturelle des élites coloniales très différente des Britanniques »(Jean-Marc Moura, 2021: 4). Dans la même perspective, "'les anciennes colonies' des Antilles françaises ou du Canada étaient des colonies d'implantation, très différentes du modèle indien britannique ou des colonies françaises en Afrique » (Ibid. : 4). C'est la raison pour laquelle, il vaut mieux « développer des modèles régionaux que des modèles globaux pour comprendre le fonctionnement de la littérature dans les contextes coloniaux et postcoloniaux » (Ibid. : 4). Cependant, même s'il faut tenir compte du contexte, il reste que les œuvres écrites dans une langue héritée de la colonisation, partagent nombre de traits liés à ce fait. On parlera en ce sens de littératures anglophones ou francophones postcoloniales et de Subaltern Studies. Celles-ci sont « étudiées dans leur dimension de résistance, de réfutation et de proposition 
de contre-discours et de formes déviantes » (Ibid. : 4). C'est dans ce contexte qu'il faut comprendre Spivak. Elle revient sur l'hypothèse selon laquelle « la production intellectuelle occidentale est, de maintes façons, complice des intérêts économiques internationaux de l'Occident» (Gayatri Spivak, 2009a : 14). Pour Spivak, l'Occident met en place sa propre histoire en réintroduisant un sujet souverain qui est pour elle géopolitiquement déterminé. Il est question pour elle de redonner la parole aux subalternes afin qu'immerge leur voix. Dès lors nous pouvons nous interroger: quel est l'enjeu d'une philosophie qui milite pour l'acentrique épistémologique ? Cette nouvelle orientation du discours ne permetelle pas de sortir des concepts monolithiques utilisés dans les sciences sociales pour parler des subalternes ? La méthode immanentiste nous permettra d'analyser ces interrogations.

\section{Spivak et les philosophes de la différence}

Spivak souligne que Jacques Derrida est le seul philosophe qui offre tous les éléments nécessaires pour démystifier «la logique assimilationniste et impérialiste de l'Occident » (Francesco Fistetti, 2009 : 50). Derrida se sert de la déconstruction pour démystifier cette logique. Notons que « la déconstruction, c'est aussi une manière d'écrire et d'avancer un autre texte » (Denis Huisman, 1996: 392). La déconstruction derridienne a pour objectif d'analyser les échafaudages ontologiques que la philosophie a érigés tout au long de son histoire. La déconstruction dans ce sens désigne l'ensemble des techniques et stratégies utilisées par Jacques Derrida pour déstabiliser, fissurer, déplacer les textes. L'objectif ici est de «différer en permanence l'aboutissement du sens, pour se maintenir dans l'espacement d'une interrogation tournoyante, où le même diffère sans cesse du même, sans pouvoir prévoir son arrivée, sa destination » (Grégoire 
Biyogo, 2005 : 67). Toutefois, déconstruire n'est pas détruire et la déconstruction s'effectue en deux temps. Elle consiste tout d'abord à un point de renversement du dualisme. Dans la phase de renversement, Jacques Derrida pose «[...] le primat transcendantal du signe par rapport à la signification » (Jürgen Habermas, 1988 : 203). Autrement dit, «celle-ci efface le signe en le dérivant, annule la reproduction et la représentation en faisant la modification survenant à une présence simple » (Jacques Derrida, 1967b : 57). La phase de renversement pour Jacques Derrida consiste «à retrouver l'originalité et le caractère non dérivé du signe contre la métaphysique classique » (Ibid.: 57). Mais un simple renversement n'opère qu'un changement de métaphysique, sans pourtant modifier le dualisme. Un déplacement à l'intérieur du cadre logocentrique ne permet pas pour autant son abolition. Loin de s'arrêter au stade du renversement, la déconstruction dans son schéma, entend aller au-delà de ces oppositions qui gouvernent les hiérarchies philosophiques, d'où sa neutralisation. Le schéma de neutralisation permet de sortir de toute situation de hiérarchie. Le terme valorisé à la phase de renversement devient neutre. Jacques Derrida développe donc une pratique aporétique de la pensée : les différences ne se résolvent pas dans une synthèse, elles fonctionnent ensemble en tant que différences sans être rapportées à une unité synthétique. Une telle approche donne au catachrèse toute son importance. En empruntant à Derrida cette notion, Spivak pense que les mots comme nationalité, souveraineté, citoyenneté sont des instances catachrétiques. Celles-ci sont des concepts surdéterminés dont le réfèrent rompt avec le centre (Gayatri Spivak, 1993 : 13). Spivak s'intéresse au «blanc textuel» de Derrida. Ce blanc a pour vocation de renverser l'institution du sujet occidental. Si l'option déconstructrice derridienne est privilégiée par Spivak, il reste qu'elle s'oppose à Michel Foucault et à Gilles Deleuze. En effet, ces deux auteurs « ont élaboré les instruments épistémologiques les plus tranchants de décentrement mais ils n'ont 
pas perçu tous les pièges de la rationalité occidentale » (Francesco Fistetti, 2009 : 50-51). Pour l'auteur de Nationalisme et Imagination, les deux penseurs sont tombés dans les «banalités rhétoriques », ce d'autant plus « qu'entre la fin des années 1960 et le début des années 1970 ils se laissent séduire par le maoïsme d'une certaine gauche européenne et par les mouvements de contestation de l'époque (étudiants, détenus, ...)» (Ibid. : 51). Or, Spivak critique le spontanéisme - d'aucuns diraient le maoïsme - des subalternistes, qui consiste à considérer que le subalterne a la capacité d'exprimer son moi de façon immédiate à travers la parole (Jean-Loup Amselle, 2008 : 155). Précisons qu'il y a selon « elle dans cette conception un phonocentrisme de la voix qui oublie que, à travers la voix du paysan soumis au régime de la tradition orale, ce n'est pas seulement une pure oralité qui s'exprime mais tout autant une marque et une présence de 1'écrit» (Ibid. : 155). Aussi, se focaliser sur cette approche, c'est manquer la voix proprement dite des subalternes. Spivak rompt ainsi radicalement avec «la vision angélique que les subalternistes ont du peuple et annonce les critiques ultérieures relatives à l'autonomie supposée des subalternes par rapport à l'élite » (Ibid. : 155).

Reprenons-nous. Il est clair que Spivak ne conteste ni l'intérêt de Foucault et Deleuze pour les opprimés. Elle dénonce simplement «l'attitude de fétichisation du concret qui les pousse à considérer le 'maoïsme' et les nouvelles luttes sociales comme 'deux sujets - en révolution - monolithiques et anonymes » (Ibid. : 51). Spivak critique ces auteurs pour leur «prétention à incarner la 'conscience' et la 'voix'-1'éloquence des masses » (Ibid. : 51). Il est donc facile de retrouver dans la formulation de ces auteurs « l'illusion cachée du sujet souverain» (Ibid. : 51). En outre, Foucault et Deleuze «ne font aucune référence ni à la division internationale du travail ni aux caractéristiques 
particulières du capitalisme global et des relations centre/périphérie ou Nord/Sud » (Ibid. : 51).

D’une manière générale, « pour Foucault et Deleuze, il semble n'exister aucun problème de "représentation" » car les deux « réduisent la théorie à un simple "relais de la pratique" qui permet aux opprimés de parler par eux-mêmes ou via la transparence du discours des intellectuels » (Ibid.. : 53). Pour Spivak, il s'agit d'une illusion. Cette illusion épistémologique est le résultat d'un réalisme positiviste, qui réintroduit le sujet constitutif pour deux raisons : « D'un côté, le Sujet du désir et du pouvoir devient «une présupposition méthodologique irréductible » (Ibid. : 53). De l'autre, « l'opprimé est hypostasié comme un "sujet proche de soi $[\ldots]$ sinon identique à soi ». Spivak conclut que Foucault et Deleuze ne sont pas « en mesure d"“imaginer" le type de désir et de pouvoir qui habitent “le sujet innommé de l'Autre de l'Europe» (Ibid. : 53). Ces deux auteurs «s'avèrent incapables de saisir "la violence épistémique" au cœur du projet occidental, de ce projet impérialiste qui vise "la constitution du sujet colonial comme Autre » (Ibid. : 53). Pour un auteur comme Deleuze, seuls ceux qui peuvent parler pour eux et réclamer, peuvent enclencher les changements révolutionnaires et, par conséquent, sortir du pouvoir. Dans cette perspective, les opprimés sont capables de contre-discours, ce qui remet en question le pouvoir. Contre la totalisation du pouvoir, Spivak pense que la solution n'est pas de rentrer dans ce même jeu de totalisation, tel qu'effectué par Deleuze et Foucault mais de contrer le pouvoir localement et, ce, de manière discontinue.

Globalement, Spivak prend ses distances avec Foucault et Deleuze car, ils sont coupables de n'accorder aucune place dans leur œuvre à l'impérialisme occidental (Jean-Loup Amselle, 2008: 143). Elle pense que ces deux 
représentants de la French Theory ont une vision eurocentrée et ne s'intéressent qu'aux opprimés du « premier monde » (Ibid. : 144). A contrario des subalternes, ces auteurs peuvent s'exprimer en leur propre nom. À ces deux auteurs, Spivak oppose Derrida. Ce dernier a pu écarter le logocentrisme et le phonocentrisme à travers la déconstruction et la différance. La différance chez Derrida se présente comme un concept économique désignant la production du différer, au double sens de ce mot. «La différance tout court serait plus « originaire», mais on ne pourra plus l'appeler «origine» ni «fondement», ces notions appartenant essentiellement à l'histoire de l'onto-théologie, c'est-à-dire au système fonctionnant comme effacement de la différence » (Jacques Derrida, 1967a : 38). La différance a l'avantage ici de reconnaitre pleinement la voix des autres. De ce fait, la « différance » est un effet et non l'originaire, elle est un effet qui ne peut, ni ne doit se faire passer pour l'original. C'est la raison pour laquelle, la « différance » se donne en tant que contaminée et tient compte de l'espace. Par conséquent, il n'y a que des suppléments de significations. On pourrait dire donc avec Spivak que, parler, écouter exigent un certain environnement matériel et intellectuel. Ces modalités font défaut aux subalternes. Il faut donc une réécriture de l'impulsion structurale utopique qui fait « délirer la voix intérieure qui est la voix de l'autre en nous ». L'enjeu de la pensée de Derrida aux yeux de Spivak est de « mettre en lumière le caractère inaudible de la voix de la femme subalterne parce qu'elle est à la fois féminine et subalterne, c'est-à-dire réduite au silence, féminine, étouffée par le discours colonial » (Jean-Loup Amselle, 2008 : 144).

\section{Spivak et les inaudibles}

L'objectif de Spivak est d'établir « la "vraie connaissance du subalterne et de sa conscience ». Celle-ci est toujours « dangereuse » (Gayatri Spivak, 2002 : 
132). Spivak soutient que «la mentalité des paysans indiens est imprégnée de phonocentrisme, dans la mesure où ils considèrent que "ce que l'on entend" d'une tradition (sruti) est doué d'une autorité supérieure » de même que «la mentalité de l'historien "est influencée par le phonocentrisme de la linguistique occidentale" »(Ibid. : 129). Par conséquent, la voix, expression parlée par excellence, peut être trompeuse. Elle «n'exprime pas "une voix-conscience unique ». Elle est « quelque chose de primordialement [...] errant, en circulation permanente et sans source reconnaissable et vérifiable» (Francesco Fistetti, 2009 : 54-55). Il faut donc, d'après Spivak, « se méfier de tout essentialisme, de toute recherche des origines pures, de toute prétention à capturer la pureté de la “voix" du subalterne» (Ibid.: 55). Cette capture de la voix de l'autre peut engendrer une certaine ambiguïté. L'exemple de cette ambigüité est celui « de la législation anglaise introduite en Inde à l'époque coloniale qui a "libéré" la veuve de l'ancien rituel hindou du sacrifice du bûcher à la mort de son mari » (Ibid. : 66). Notons que «l'interdiction, par les Anglais, de ce rituel a été généralement interprétée comme un exemple typique du cliché occidentaliste ». Ce cliché stipule que «les hommes blancs sauvent les femmes foncées des mains d'hommes foncés » (Ibid. : 67). L'objectif de ce cliché est : « rendre la dignité et l'autonomie aux femmes subalternes exige d'appliquer les paramètres culturels de la modernité occidentale » (Ibid. : 67). Pour s'en convaincre, Spivak prend l'exemple de la sati. Elle montre que la signification de la sati « est devenue incompréhensible puisqu'elle a été réduite au phénomène de l'immolation de la veuve et, à ce titre, assimilée à une coutume barbare et donc à un crime, par les Britanniques » (Jean-Loup Amselle, 2008 : 144). Il serait donc «suffisant de transformer en "crime" - et d'intégrer dans un article du Code Pénal - ce qui était auparavant considéré comme un rituel consacré par la tradition religieuse hindoue ou, au moins par certaines de ses versions » (Francesco Fistetti, 2009 : 
67). Dans cette logique, «l'illusion consiste à croire qu'il suffit de concevoir la femme du Tiers-Monde comme sujet des droits humains pour l'arracher à sa subalternité » (Ibid. : 67). Cette illustration de Spivak met en relief l'échec ou la suppression de l'espace de parole pour la femme subalterne. Elle affirme dans ce sens : «la subalterne ne peut parler. Il n'y a aucune vertu à une liste globale incluant pieusement l'élément «femme». La représentation n'a pas disparu [wither away]. L'intellectuelle femme, en tant qu'intellectuelle, à une tâche qu'elle ne doit pas désavouer d'un grand geste » (Gayatri Spivak, 2009a : 103). À travers l'exemple du Sati, Spivak montre qu'il s'agit d'une situation d'effacement du sujet subalterne et opprimé par les colonisateurs. Il s'agit d'« un exemple paradigmatique de la "violence épistémique" de l'impérialisme qui, dans le cas de la différence sexuelle, finit par en effacer doublement la trace » (Francesco Fistetti, 2009 : 67). Les conséquences de cet effacement peuvent se résumer par le fait que «la femme est niée et occultée à la fois dans la culture prémoderne du patriarcat et comme objet de la culture hypermoderne de l'impérialisme » (Ibid. : 67). Elle ajoute plus loin : «si, dans le contexte de la production coloniale, les subalternes n'ont pas d'histoire et ne peuvent pas parler, les subalternes en tant que femmes sont encore plus profondément dans l'ombre » (Gayatri Spivak, 2009a: 53). Dans le cas de la sati, «il s'agit pour elle de redécouvrir sa signification, en la "lisant", à la manière d'un palimpseste » (JeanLoup Amselle, 2008 : 144), c'est-à-dire «à travers des phénomènes récents du même ordre » (Ibid. : 144). À partir de là, il est question pour Spivak d'enrichir la théorie subalterniste, en précisant les conditions d'accès à la voix inaudible des subalternes. Laisser parler, écouter mais surtout entendre, consiste d'abord à ne pas parler à la place des autres. Les subalternes ne peuvent pas parler, y montret-elle, à rebours d'un certain nombre d'autres positionnements intellectuels. Alors pour qu'une parole soit entendue par tout le monde, que faut-il alors ? Pour 
répondre à cette interrogation, Spivak prend, dans un autre entretien, l'exemple d'un autre cas de suicide ; a contrario de cette jeune femme des années 1920, l'acte par lequel Mohamed Bouazizi s'immole par le feu, le 17 décembre 2010, est compris comme un acte politique au point de déclencher ce qu'on appellera la révolution tunisienne puis, par extension, le printemps arabe (Anne Versjus, 2015). Pour qu'advienne une telle écoute, une telle réception de cet acte de parole radical, il a fallu, précise Spivak, qu'il soit entendu par une infrastructure politique préexistante. Et celle-ci, du fait de sa capacité d'écoute, a été ensuite en mesure de lui faire écho, de le compléter et finalement de le prolonger à travers des actes révolutionnaires dont « le rayon d'action a dépassé, tout en l'englobant, la seule catégorie de population que l'on peut définir par le terme subalterne » (Jonathan Chauveau, 2011).

L'auteur de Les subalternes peuvent-elles parler s'intéresse ici « davantage à ce qui est écrit et qui passe inaperçu, qui n'est pas lu dans les nombreuses littératures indiennes » (Gayatri Spivak, 2009b: 66). Ainsi par exemple, lorsqu'une femme accomplit un acte de résistance en l'absence d'une infrastructure qui nous permette de reconnaitre ce qu'est la résistance, sa résistance est vaine (Ibid. : 77). Une telle logique lui fait dire que « le subalterne est quelqu'un qui n'a pas accès à la mobilité sociale». Aussi ne s'agit-il pas simplement « de s'efforcer d'entendre et de comprendre la subalternité » (Ibid. : 81), mais il «s'agit vraiment de gagner le droit, qui requiert beaucoup d'efforts, d'être dans la normalité subalterne de façon à pouvoir intervenir en elle et vous y faire entendre» (Ibid.: 81). Le subalterne doit «entrer dans l'espace hégémonique pour y être oppositionnel » (Ibid. : 81). Il doit se battre afin que ses droits soient restaurés. 
Pour rappel, par subalternes, Spivak entend ceux qui «n'ont pas d'accès aux lignes abstraites de la mobilité sociale, même après avoir obtenu une victoire légale en leur nom » (Ibid. : 81). La « subalternité » renvoie finalement à une « idée pratique d'absence d'accès» (Ibid.: 88), ce d'autant plus que «les femmes et les hommes sont dans une situation inégale face à la justice distributive » (Ibid. : 88). La « subalternité n'est donc pas une chose pathétique à propos des gens subalternes ». C'est «la description d'une position politique ou sociale» (Ibid. : 88). Spivak veut que les «subalternes parlent pour euxmêmes » (Ibid. : 88), car ils « ont autant de vie intérieure que n'importe qui » (Ibid. : 88). Ce «n'est pas non plus qu'ils soient des gens incapables de sentiment, ou qui en auraient moins que les autres » (Ibid. : 88-89). Le problème du subalterne peut se résumer au fait « qu'ils n'ont pas accès à la sphère publique pour que leur résistance puisse être reconnue comme telle » (Ibid. : 89).

\section{Les conditions de la reconnaissance des subalternes}

Pour Spivak, «les subalternes ne peuvent parler qu'à condition de les insérer, en tant que subalternes, dans le "circuit de l'hégémonie" et de leur dévoiler ses codes. C'est-à-dire, de leur donner accès à une instruction qui ne soit pas de bas étage. Spivak fait référence ainsi à l'éducation. C'est le sens de son activisme en tant que formatrice exigeante d'enseignants d'écoles élémentaires dans l'ouest du Bengale. "Enseigner, dit-elle, c'est opérer un changement en restant ancrés dans un espace commun » (Gayatri Spivak, 2012). Au fond, les exemples sont légion. Les marginalisés se sont souvent organisés pour défendre les intérêts de leur classe sociale. Elle pense aux femmes qui, au cours du XIX ${ }^{\mathrm{e}}$ siècle en Europe, sont passées de la condition d'épouses et de mères adhérant au discours conjugaliste de la différence complémentaire, à la position de femmes 
conscientes de leur droit aux droits et revendiquant l'égalité jusque-là négligée. Pour penser la "différence naturelle" en termes d'inégalités à combattre, il a fallu tout un siècle au cours duquel l'éducation des filles est peu à peu devenue un enjeu politique. Pour qu'advienne le féminisme et, avec lui, la conscience de classe de sexe liée aux êtres de couleurs, il a donc bien fallu que se mettent en place de nouvelles conditions matérielles et intellectuelles permettant de faire émerger cette conscience chez les femmes et de la rendre audible et recevable dans l'espace public. À travers la déconstruction derridienne, elle invite à rompre avec toute forme d'essentialisme et d'expressivisme. Elle applique cette démarche à l'analyse critique du projet impérialiste de l'Occident sur le reste du monde (Francesco Fistetti, 2009 : 58).

\section{Conclusion}

Les subalternes peuvent-elles parler? est une philosophie dont l'objectif est de rendre visibles les subalternes. Le subalterne, concept inspiré par Antonio Gramsci, renvoie aux sans-voix, mieux à celui dont la voix, la parole ne compte pas. Que ça soit la femme, le fou, le vieux, le prisonnier, que ça soit l'immigré, c'est un même droit qui leur est dénié. Cette négation ne consiste pas au droit à l'expression car tous parlent (Aurélien Berthier, 2013), mais bien au droit d'être

entendu, écouté et participer activement à l'espace publique. À la question, les subalternes peuvent-elles parler? Spivak répondra donc par la négative. Les subalternes ne peuvent pas parler en raison même de leur position de subalternité. Ceux qui prétendent les entendre ou les écouter ne font en réalité que parler à leur place, le but étant de sauvegarder leurs intérêts. Pour pallier cette situation, Spivak accorde à l'éducation une place prépondérante. Les subalternes, pour Gayatri Spivak, ne peuvent parler qu'à condition de les insérer, en tant que 
subalternes, dans le circuit de l'hégémonie. Ce circuit est en réalité la classe dominante. Les insérer dans cet espace, permettra à coup sûr aux subalternes de défendre leurs intérêts. En outre, les insérer dans ce circuit, exige par conséquent de leur dévoiler les codes, c'est-à-dire, de leur donner accès à une instruction qui ne soit pas médiocre. C'est dans ce sens qu'elle affirme qu' « enseigner, c'est opérer un changement en restant ancrés dans un espace commun » (Spivak, 2012b). 


\section{Bibliographie}

Berthier, Aurélien, «Qu'est-ce que la dimension postcoloniale ? , Agir par la culture, $\mathrm{n}^{\circ} 33,2013$, pp. 10-25.

Butler, Judith, Ces Corps qui comptent. De la matérialité et des limites discursives du « sexe », New York, Amsterdam, 2009.

Coulibaly, Bassidiki, Du Crime d'être "noir". Un Milliard de "Noir" dans une prison identitaire, Paris, Homnisphères, 2006.

Derrida, Jacques, De la grammatologie, Paris, Minuit, 1967a.

Derrida, Jacques, La Dissémination, Paris, Seuil, 1972.

Derrida, Jacques, La Voix et le phénomène, Paris, P.U.F, $1967 \mathrm{~b}$.

Derrida, Jacques, L'Écriture et la différence, Paris, Seuil, 1967c.

Derrida, Jacques, Marges, Paris, Minuit, 1972.

Feyerabend, Michel, Contre la méthode : une archéologie des sciences humaines, Paris, Seuil, 1973.

Fistetti, Francesco, Théories du multiculturalisme. Un parcours entre philosophie et sciences sociales, Paris, La Découverte, 2009.

Habermas, Jürgen, Le Discours philosophique de la modernité, Paris, Gallimard, 1988.

Hardt, Michael; Negri, Antonio, Empire, Paris, Exils, 2000.

Hottois, Gilbert, L'Inflation du langage dans la philosophie contemporaine, Bruxelles, Université de Bruxelles, 1979.

Jean-Clet, Martin, Derrida - Un Démantèlement de l'Occident, Paris, Max Milo, 2013.

Kofman, Sarah, Lectures de Derrida, Paris, Galilée, 1984. 
Mamadou, Diouf, L'Historiographie indienne en débat. Colonialisme, nationalisme et sociétés postcoloniales, Paris, Karthala-Sephis, 1999.

Nkolo Foe, Le Postmodernisme et le nouvel esprit du capitalisme sur une philosophie globale d'Empire, Dakar, Conseil pour le développement de la recherche en sciences sociale en Afrique, 2008.

Nietzsche, Friedrich, La Naissance de la tragédie, Paris, Gallimard, 1997.

Rorty, Richard, L'Homme Spéculaire, Paris, Seuil, 1990.

Saint Augustin, De Quantitate anima, XIII, 22, Schoningh, Paderbon, 1968.

Silvano Petrosino, Jacques Derrida et la loi du possible, Paris, Cerf, 1994.

Spivak, Gayatri Chakravorty, Nationalisme et imagination, Paris, Payot et Rivages, 2011.

Spivak, Gayatri Chakravorty, En d'autres mondes, en d'autres mots. Essais de politique culturelle, Paris, Payot, 2009b.

Spivak, Gayatri Chakravorty, Les Subalternes peuvent-elles parler? Paris, Amsterdam, 2009a.

Spivak, Gayatri Chakravorty, «In a word: interview », Outside in the Teaching Machine, New York-London, Routledge.

Spivak, Gayatri Chakravorty, «C'est la volonté d'entendre mon ancêtre qui m'a engagée sur cette voie », Philosophie Magazine, 28 juin 2012.

Steinmetz, Rudy, Les Styles de Derrida, Bruxelles, De Boeck, 1994.

Trigano, Shmuel, La Nouvelle idéologie dominante. Le post-modernisme, Paris, Hermann, 2012.

Tremblay, Pierre-André, Compte-rendu de Roland Lardinois (textes réunis et présentés par), Miroir de l'Inde, Études indiennes en sciences sociales, Paris, MSH, 1989. 